\title{
HOLOMORPHIC VECTOR FIELDS AND THE FIRST CHERN CLASS OF A HODGE MANIFOLD
}

\author{
YOZO MATSUSHIMA
}

In a recent paper [2] Bott has proved that if a connected compact complex manifold admits a nonvanishing holomorphic vector field, then all the Chern numbers of $M$ vanish.

In this paper we first prove the following theorem.

Theorem 1. Let $M$ be a connected Hodge manifold, and suppose that there exists a nonvanishing holomorphic vector field $X$ in $M$. Then there exists a nonvanishing holomorphic 1-form $\omega$ in $M$ such that $\omega(X) \neq 0$. In particular, the first Betti number $b_{1}(M)$ of $M$ is different from zero.

We shall then study the structure of a Hodge manifold with zero first Chern class. We denote by $c_{1}(M)$ and $q(M)$ the first Chern class and the irregularity (i.e., one half of the first Betti number) of $M$ respectively, and by $G$ the identity component of the group of all holomorphic transformations of $M$. The group $G$ is a connected complex Lie group.

We shall prove the following two theorems which sharpen some of the recent results of Lichnerowicz [5].

Theorem 2. Let $M$ be a connected Hodge manifold such that $c_{1}(M)=0$. Then the group $G$ is an abelian variety of dimension $q(M)$ and the isotropy subgroup of $G$ at any point in $M$ is a finite group.

Theorem 3. Let $M$ be a connected Hodge manifold and assume that $c_{1}(M)$ $=0$ and $q(M)>0$. Then there exist an abelian variety $A$ and a connected Hodge manifold $F$ with the following properties.

a) $c_{1}(F)=0$ and $q(F)=0$;

b) $A \times F$ is a finite regular covering space of $M$ and the group of covering transformations is solvable.

After having finished this work, the author learned that Calabi stated these two theorems in his paper [4] as his well-known conjecture, and proved them under the assumption that $M$ is a connected compact Kähler manifold with vanishing Ricci curvature tensor.

1. Let $M$ be a connected compact Kähler manifold, and $\mathfrak{h}$ and $g$ denote, respectively, the complex vector space of all holomorphic 1-forms and the complex Lie algebra of all holomorphic vector fields in $M$. Then $\operatorname{dim} \mathfrak{h}=q(M)$ and we can identify $g$ with the Lie algebra of the group $G$. If $\omega \in \mathfrak{h}$ and $X \in \mathfrak{g}$, then

Communicated November 27, 1968 and, in revised form, October 27, 1969. 
$\omega(X)$ is a holomorphic function on $M$ and hence a constant. Therefore $(\omega, X)$ $\rightarrow \omega(X)$ defines a bilinear form $B$ on $\mathfrak{h} \times \mathfrak{g}$.

Now let $\alpha$ be the canonical holomorphic mapping of $M$ into the Albanese variety $A(M)$ of $M[1]$, [6]. There exists also a complex Lie group homomorphism $\hat{\alpha}$ of $G$ into the complex torus $A(M)$ such that $\alpha(\varphi x)=\hat{\alpha}(\varphi) \alpha(x)$ for any $\varphi \in G$ and $x \in M$. Let $I$ be the kernel of the homomorphism $\hat{\alpha}: G \rightarrow A(M)$, and $I^{0}$ the identity component of $I$. The subalgebra $i$ of $g$ corresponding to $I$ consists of all holomorphic vector fields $X$ in $M$ such that $\omega(X)=0$ for all $\omega \in \mathfrak{h}$. In particular, if zero $(X), X \in \mathrm{g}$, is non-empty, then $X \in \mathfrak{i}$, where $z e r o(X)$ denotes the set of zero points of $X$. Assume now that $M$ is a Hodge manifold, and let $\varphi: M \rightarrow P^{N}$ be a projective imbedding of $M$ into a complex projective space $P^{v}$. Let $G_{\varphi}$ be the group of all holomorphic transformations of $M$ induced by the projective transformations of the ambient space $P^{v}$ which leave stable the submanifold $M$. The subalgebra $g_{\varphi}$ of $g$ corresponding to $G_{\varphi}$ consists of the restriction in $M$ of all holomorphoic vector fields in $P^{N}$ tangent to $M$. By a fixed point theorem of Borel [3], every $X \in g_{\varphi}$ has a zero point and hence $g_{\varphi} \subset \mathfrak{i}$ for any projective imbedding $\varphi$. On the other hand, a theorem of Blanchard [1, Theoreme principal I] asserts that there exists a projective imbedding $\varphi_{0}$ such that $I \subset G_{\varphi_{0}}$. It follows from these that 1) $\mathfrak{i}=g_{\varphi_{0}}$ and hence $\mathfrak{i}$ consists of all $X \in \mathfrak{g}$ such that zero $(X)$ is non-empty; 2) [I: $\left.I^{0}\right]<\infty$, because $I^{0}$ coincides with the identity component $G_{\varphi_{0}}^{0}$ of $G_{\varphi_{0}}$ and, since $G_{\varphi_{0}}$ is an algebraic group, we have $\left[G_{\varphi_{0}}: G_{\varphi_{0}}^{0}\right]<\infty$. We have thus proved

Proposition 1. Let $M$ be a connected Hodge manifold, and I the kernel of the homomorphism $\hat{\alpha}: G \rightarrow A(M)$. Then the number of connected components of $I$ is finite, and the Lie algebra $\mathrm{i}$ of $I$ consists of all holomorphic vector fields $X$ in $M$ such that zero $(X)$ is non-empty.

Now let $X$ be a nonvanishing holomorphic vector field in $M$. Then $X$ does not belong to $i$ by Proposition 1, and there exists a holomorphic 1-form $\omega$ such that $\omega(X) \neq 0$, because $i$ consists of all $Y \in g$ such that $\omega(Y)=0$ for all $\omega \in \mathfrak{h}$. Since $\omega(X) \neq 0, \omega$ is nonvanishing, which proves Theorem 1 .

Remark. Let $M$ be an even-dimensional connected compact semi-simple Lie group. Then there exists a left invariant complex structure on $M$, a right invariant vector field in $M$ is a nonvanishing holomorphic vector field, and the first Betti number of $M$ is zero. This example shows that the existence of a nonvanishing holomorphic vector field does not necessarily imply the nonvanishing of the first Betti number of a connected compact complex manifold. However, in this example, for any right invariant vector field $X$ there exists a right invariant 1 -form $\omega$ such that $\omega(X) \neq 0$, and $\omega$ is holomorphic although $\omega$ is not a closed form.

2. Let $M$ be a connected compact Kähler manifold such that $c_{1}(M)=0$. Then by a theorem of Lichnerowicz $[5, a]$ the bilinear form $B: \mathfrak{h} \times \mathfrak{g} \rightarrow C$ is nondegenerate. In particular, we have $\operatorname{dim} g=\operatorname{dim} \mathfrak{q}=q(M)$ and every non-zero holomorphic vector field in $M$ has no zero point. Hence from Proposition 1 we 
obtain the following

Proposition 2. Let $M$ be a connected Hodge manifold such that $c_{1}(M)=0$. Then the homomorphism $\hat{\alpha}: G \rightarrow A(M)$ is an isogeny, that is, a surjective homomorphism with a finite kernel $I$. In particular, $G$ is an abelian variety of dimension $q(M)$. If $\varphi: M \rightarrow P^{N}$ is a projective imbedding, then the group $G_{\varphi}$ of holomorphic transformations of $M$ induced by the projective transformations of $P^{N}$ is finite.

The assertion of Theorem 2 is included in Propositions 1 and 2.

3. Let $M$ be a connected Hodge manifold such that $c_{1}(M)=0$ and let $M_{1}$ $=\alpha^{-1}(e)$, where $\alpha: M \rightarrow A(M)$ is the canonical holomorphic mapping and $e$ denotes the identity element of the torus $A(M)$. From the universality of the mapping $\alpha$, we can easily conclude that $M_{1}$ is connected $[5, b]$ and see that $c_{1}\left(M_{1}\right)=0$. Since the finite group $I$ acts on $M_{1}$, let $E$ be the holomorphic fibre bundle over $A(M)$ with fibre $M_{1}$ associated with the holomorphic principal bundle $0 \rightarrow I \rightarrow G \rightarrow A(M) \rightarrow 0$. Then $E$ is the quotient of $G \times M_{1}$ by the action of $I$ defined by $\phi(\varphi, u)=\left(\varphi \cdot \psi^{-1}, \phi(u)\right)\left(\phi \in I, \varphi \in G, u \in M_{1}\right)$. Let $\beta$ be the holomorphic mapping of $G \times M_{1}$ into $M$ defined by $\beta(\varphi, u)=\varphi(u)$. Then it is easy to see that $\not{\beta}$ is surjective and that $\beta$ induces a bijective holomorphic mapping of $E$ onto $M$ such that the diagram

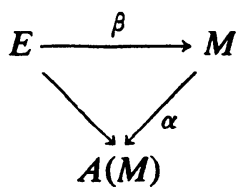

is commutative. Thus, $M$ is a fibre bundle over $A(M)$ with projection $\alpha$ and fibre $M_{1}$. It follows also from the above that $G \times M_{1}$ is a finite covering space of $M$ with $I$ as the group of covering transformations. If $q\left(M_{1}\right)=0$, then we have completed the proof of Theorem 3, because $I$ is abelian. Assume $q\left(M_{1}\right)>0$. Then we can find a connected Hodge manifold $M_{2}$ such that $c_{1}\left(M_{2}\right)$ $=0$ and that $G_{1} \times M_{2}$ is a covering space of $M_{1}$ with a finite abelian covering transformation group, where $G_{1}$ denotes the identity component of the group of holomorphic transformations of $M_{1}$. Continuing in this way we get a sequence $\left\{M_{i}\right\}$ such that $c_{1}\left(M_{i}\right)=0, \operatorname{dim} M_{i+1}=\operatorname{dim} M_{i}-q\left(M_{i}\right)$ for $i=0,1, \cdots$, where $M_{0}=M$. Therefore, there must exist an integer $k$ such that $q\left(M_{k}\right)=0$ (the dimension of $M_{k}$ might be zero). Let $A=G \times G_{1} \times \cdots \times G_{k-1}$ and $F$ $=M_{k}$. Then $A$ is an abelian variety and $A \times F$ is a covering manifold of $M$ with a finite solvable covering transformation group. Hence Theorem 3 is proved.

\section{References}

[1] A. Blanchard, Sur les variétés analytiques complexes, Ann. Ecole Norm. Sup. 73 (1956) 157-202.

[2] R. Bott, Vector fields and characteristic numbers, Michigan Math. J. 14 (1967) 231255. 
[ 3 ] A. Borel, Groupes linéaires algébriques, Ann. of Math. J. 64 (1956) 20-82.

[ 4 ] E. Calabi, On Kähler manifolds with vanishing canonical class, Algberaic geometry and topology, A symposium in honor of S. Lefschetz, Princeton University Press, Princeton, 1957, 78-89.

[ 5 ] A. Lichnerowicz, a) Variétés kählériennes et première classe de Chern, J. Differential Geometry 1 (1967) 195-224, b) Variétés kählériennes à première classe de Chern positive ou nulle, C. R. Acad. Sci. Paris 268 (1969) 876-880.

[6] A. Weil, On Picard varieties, Amer. J. Math. 74 (1952) 865-894.

UNIVERSity OF Notre DAME 